\title{
The Biophysical Modeling of the Transport Phenomena in the Living Systems
}

\author{
Janos Vincze, Gabriella Vincze-Tiszay \\ Health Human International Enviroment Foundation, Budapest, Hungary \\ ndp@t-online.hu
}

\begin{abstract}
This article describes the principles of biological transport. The transport phenomena mean the variation in time and space of generalized forces when they generate flows for which conservation laws apply. After we describes: mass-, impulse-, energy- and electric-charge-transport and their mathematical characteristic equations. It emphasizes the physical aspects of transport and examines the assumptions and concepts underlying the equations most widely used to characterize transport. In the living organisms, flows are not generated only by the conjugated generalized forces, but also by the simultaneous action of other forces, so appears the cross-effects. The biophysical modeling offer a „language” of quantitative and qualitative processsing of experimental data, being compatible and adequate to the laws of biology.
\end{abstract}

Key words: transport phenomena, biophysical modeling, diffusion, viscosity, thermal conductibility, electric conductibility, cross-effects

\section{Introduction}

The modelling method in biophysics consists of the creation of certain devices (models), with which processes analogue with those happening in living organism are studied. The biophysical model though abstract reasoning leads to models of the phenomena which by simplifying and isolating some aspects of the phenomena, discover laws and relationships which describe with a certain approximation the behaviour or functioning of bodies or biological ensembles.

The biophysical models offer a „language” of quantitative and qualitative processing of experimental data, being compatible and adequate to the laws of biology.

Homeostasis is the ability to maintain the structure and functions of the living organism within optimal limits, on the basis of the adaptive requirements imposed by the surrounding environment. [1] This implies complex control and anticipation mechanisms through which the organism and its constitutive systems autoadjustmets the equilibrium level for the given situation, dynamic equilibrium between the disturbing and compensating factors. Homeostasis is a dynamic process of permanent adaptation (by the transport phenomena), each of its sequences representing a new equilibrium which, in the following sequence, will be at a different level, in accordance with the requirement, between the permissive limits of the systems reactivity. 


\section{Definition of the transport phenomena}

The transport phenomena mean the variation in time and space of generalized forces when they generate flows for which conservation laws apply. [2]

This general and strongly scientific definition of the transportation phenomena has two major merits: 1) particular forms of transportation can be deducted from it (mass transport diffusion; energy transport - thermal conductibility; impulse transport - viscosity; electric charge transport - electric conductibility, crossed effects and other); 2.) it allows a quantitative characterization of the product exchange, which was impossible based on the previous definitions.

If $\mathbf{W}$ - the amount of the transported parameter, for which the conservation law is valid; $\mathbf{K}-\mathbf{a}$ constant dependent on the type of transportation and the nature of the transported parameter; grad a - the generalized force, then the amount of the parameter (flow) transported through the surface $\mathbf{d S}$ in the dt time frame will be given by the relation:

$$
\mathrm{W}=\mathrm{K} \int_{t_{1}}^{t_{2}} \iiint_{S(x, y, z)} \operatorname{grad} a d S d t
$$

If the transportation takes place only after a direction $\mathbf{x}$, then we obtain the formula:

$$
\mathrm{W}=\mathrm{K} \int_{t_{1}}^{t_{2}} \int_{x_{1}}^{x_{2}} \operatorname{grad} \mathrm{a}_{\mathrm{x}} \mathrm{dx} \mathrm{dt}
$$

The differential form is the following:

$$
\partial W=K \cdot \frac{\partial a}{\partial x} \cdot \Delta S \cdot \Delta t
$$

Making the proper replacements in the relation above, we obtain the classical laws which describe particular, simple transportation phenomena.

With non stationary transportation we understand those transportations where the value of the flow is modified in time from one point to the other. Making the right replacements in the relationship above we obtain the classical laws which describe the simple non stationary transportation phenomena.

\section{Mass transport}

According to the general definition, the diffusion is a mass transportation under the action of a generalising force, which can be the concentration gradient, the pressure gradient, etc. It is about a mass flow, so that the parameter transported complies with the mass preservation law [2, 3]. By replacing in the general relation the generalized force with the $\mathbf{d c} / \mathbf{d} \mathbf{x}$ concentration gradient the $\mathbf{K}$ constant with -D, where $\mathbf{D}$ is the diffusion coefficient through differentiation, we obtain Fick's first law:

$$
\Delta \mathrm{m}=-\mathrm{D} \frac{d c}{d x} \Delta \mathrm{S} \Delta \mathrm{t}
$$

The diffusion is explained on the basis of the spontaneous movement of the molecules which have the tendency to spread in equal numbers in each subspace tendency which arises from the concentration gradient. The minus sign in Fick's first law indicates the sense of development of the diffusion from the higher concentration towards the lower concentration. 
Starting from Fick's first law, we can study the time variation of the concentration at the level of a given section. If the gradient is constant, then through the layer of $\mathrm{dx}$ thickness of the section considered each number of molecules equal to the number of molecules which leave the layer towards the less concentrated area enters each second from the more concentrated area, hence the concentration in that layer remains constant. But if the concentration varies closer and closer on the direction $\mathbf{x}$, then in time the concentration in the various layers will also change. For the calculation of this variation in time, we must take into account the fact that the entry speed $\mathrm{dn} / \mathrm{dt}$ of the substance in the analysed layer in the $\mathbf{t}$ moment will be equal to:

$$
\frac{d n}{d t}=-\mathrm{D} \cdot \mathrm{S}\left(\frac{\partial c}{\partial x}\right)_{t} \cdot d x
$$

And the exit speed through the opposite side will differ from the first one, due to the variation of the concentration gradient:

$$
\mathrm{c}-\mathrm{dc}=\mathrm{c}-\left(\frac{\partial c}{\partial x}\right)_{t} \cdot d x
$$

Hence the exit speed will be equal to:

$$
\begin{gathered}
\frac{d n^{\prime}}{d t}=-\mathrm{D} \cdot \mathrm{S}\left(\frac{\partial(c-d c)}{\partial x}\right)_{t} \\
\frac{d n^{\prime}}{d t}=-\mathrm{D} \cdot \mathrm{S}\left[\left(\frac{\partial c}{\partial x}\right)_{t}-\left(\frac{\partial^{2} c}{\partial x^{2}}\right)_{t} \cdot d x\right]
\end{gathered}
$$

It means that the variation per second of the amount of substance from the layer of $\mathrm{dx}$ thickness is $(\mathrm{dn}-\mathrm{dn}$ ')/dt, and by dividing these values at the S.dx volume of the layer gives us the sought concentration variation:

$$
\frac{d n-d n^{\prime}}{S \cdot d x \cdot d t}=\left(\frac{\partial c}{\partial t}\right)_{x}
$$

By replacing the values of dn/dt, respectively dn'/dt we arrived to Fick's second law:

$$
\left(\frac{\partial c}{\partial t}\right)_{x}=\mathrm{D} \cdot\left(\frac{\partial^{2} c}{\partial x^{2}}\right)_{t}
$$

Which establishes that the variation in time of the concentration in a space is proportional with the variation in space of the concentration gradient in that particular timeframe. This law lays at the basis of determination of the diffusion by measuring the distribution of the concentration in the system after a determined period of time.

A particular case of diffusion is dialysis, which consists in the diffusion thought semipermeable membranes of the substances with a molecule smaller than the membranes pores. The dialysis takes place up to the equalising of the concentration in the two parts of the membrane. It is also used in the modern therapy. An example is the artificial kidney whose functioning is based on achieving an extrarenal and extracorporal dialysis which allows the purification of the organism from catabolytes and exo- and endogenous toxins because the depuration could not be performed by the non functional kidney. 


\section{Impulse transport}

According to the general definition, viscosity is an impulse transport under the action of a generalized force which is the speed gradient. [2,3]

So it is about an impulse flow, so that the transported parameter obeys to the impulse preservation law. By replacing in the general equation the generalized force with the speed gradient $\mathbf{d v} / \mathbf{d x}$, the constant $\mathbf{K}$ with $-\boldsymbol{\eta}$, where $\boldsymbol{\eta}$ is the viscosity coefficient and, through differentiation, we can deduct Newton's law:

$$
\begin{gathered}
\Delta \mathrm{p}=-\boldsymbol{\eta} \frac{d v}{d x} \Delta \mathrm{S} \Delta \mathrm{t} ; \quad \mathbf{F} \cdot \Delta \mathbf{t}=\Delta \mathbf{p} \\
\mathbf{F}=-\boldsymbol{\eta} \frac{d v}{d x} \Delta \mathrm{S}
\end{gathered}
$$

The viscosity is a fluid and gas property determined by the internal rub which starts in the fluid's mass as a consequence of the movement of the layers which move away from one another with different speeds. Due to the cohesion forces, the reciprocal sliding of the layers of liquids produces an internal resistance, at a rubbing force called viscosity.

Newton's $2^{\text {nd }}$ law is obtained by making a similar deduction as in the case of Fick's $2^{\text {nd }}$ law for the case of viscosity, from the general equation we obtain:

$$
\left(\frac{\partial v}{\partial t}\right)_{x}=\eta \cdot\left(\frac{\partial^{2} v}{\partial x^{2}}\right)_{t}
$$

Which we will call Newton's $2^{\text {nd }}$ and it also applies in the case of non stationary conditions on viscosity.

\section{Energy transport}

According to the general definition, the thermal conductibility is an energy transport under the action of a generalized force, which is the temperature gradient. [2, 3] Hence, it is about an energy flow, so that the parameter transported complies with the energy preservation law. By replacing in the general relation the generalized force with the temperature gradient $\mathrm{dT} / \mathrm{dx}$, the constant $\mathrm{K}$ with $\chi$, where $\chi$ is the thermal conductibility coefficient, through differentiations, we can deduct Fourier's law:

$$
\Delta \mathbf{Q}=-\chi \cdot \frac{d T}{d x} \cdot \mathbf{d S} \cdot \mathbf{d t}
$$

where: $\Delta \mathrm{Q}$ - the amount of energy to be transported; dS - the surface through which the transportation takes place; dt - transportation time. The shape of energy transported under the action of the temperature gradient is called caloric energy or simpler - heat.

Fourier's $2^{\text {nd }}$ law is obtained by making a similar deduction as in the case of Fick's second law, for the thermal conductibility, from the general equation we obtain:

$$
\left(\frac{\partial T}{\partial t}\right)_{x}=\chi \cdot\left(\frac{\partial^{2} T}{\partial x^{2}}\right)_{t}
$$


which we will call Fourier's $2^{\text {nd }}$ law and it applies in the case of non stationary conditions on the thermal conductibility.

\section{Electric charge transport}

According to the general definition, the electric conductibility is an electric charge transport under the action of a generalized force, which is the tension gradient. [2,3] Hence it is about an electrical charge flow, so that the transported parameter complies with the electric charge preservation law. By replacing in the general force with the temperature gradient $\mathrm{dU} / \mathrm{dx}$, the constant $\mathrm{K}$ is the electric conductibility coefficient and by subtraction we can deduce Ohm's law:

$$
d Q=-K \cdot \frac{d U}{d x} \cdot d S \cdot d t
$$

where: dQ - electrical charge; $\mathrm{K}$ - electric conductibility coefficient; dU - is the electric potential; $\mathrm{dx}$ - the pathway difference; $\mathrm{dS}$ - the surface that the transportation takes place through; $\mathrm{dt}$ - time frame.

It is well known the fact that the metals are good electric conducers. In the simplest model of the metallic conduction it is implied that each atom of a crystalline network cedes one or more of its exterior electrons. These electrons are free to move through the crystalline network, knocking at time intervals with the stationary positive ions.

Ohm's second law is obtained by making a similar deduction as in the case of Fick's second law. For the case of electric conductibility, from the general equation we will obtain:

$$
\left(\frac{\partial U}{\partial t}\right)_{x}=\mathrm{K} \cdot\left(\frac{\partial^{2} U}{\partial x^{2}}\right)_{t}
$$

which we will call Ohm's $2^{\text {nd }}$ law and it applies in the case of non stationary conditions on the electric conductibility.

In the living organisms in the first approximation we are not forced to consider the non stationary phenomena, but at a deeper characterization we must take into account at the exact calculation of the characteristic parameters. $[4,5]$

\section{Endocytosis}

Endocytosis is a mechanism by which cells internalize solutes from the extracellular fluid. [6] These solutes include nutrients, growth factors, hormones and enzymes. As can be seen from the sketch in Fig. 1. the cell membrane envelops a portion of the outside medium, eventually forming an intracellular vesicle containing extracellular material.

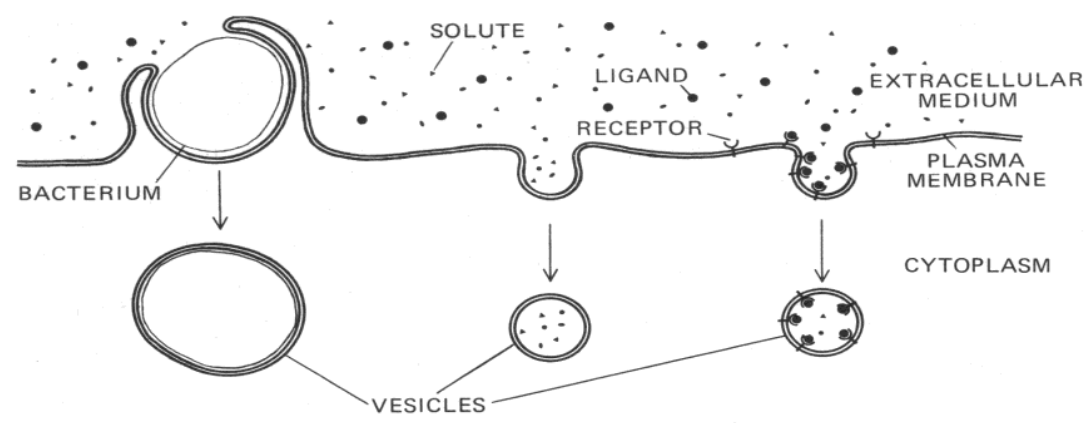

Fig. 1. Endocytosis 
Let us take $\mathrm{M}$ as the number of vesicles on the dS surface of the cellular membrane, $\mathbf{c}_{\mathbf{e}}$ and $\mathbf{c}_{\mathbf{i}}$ the concentration of the substance to be transported in the extra and intracellular environment, in which case the variation of the possibility to form the $\mathrm{n}^{\text {th }}$ vesicle according to the type is given by the equation: [6]

$$
\begin{gathered}
\mathbf{K} \cdot \frac{d P_{n}(t)}{d t}=\mathbf{c}_{\mathbf{k}} \cdot\left(\mathbf{P}_{\mathbf{n}-1}(\mathbf{t})-\mathbf{P}_{\mathbf{n}}(\mathbf{t})\right)+\mathbf{c}_{\mathbf{b}} \cdot\left(\mathbf{P}_{\mathbf{n}+1}(\mathbf{t})-\mathbf{P}_{\mathbf{n}}(\mathbf{t})\right) ; \\
\boldsymbol{1} \leq \boldsymbol{n} \leq \boldsymbol{M}
\end{gathered}
$$

The $\mathrm{n}^{\text {th }}$ vesicle can appear in two types: either there are $\mathrm{n}-1$ vesicles and after the binding of a molecule to be transported on the surface of the membrane the nth vesicle is formed, or the membrane contains $\mathbf{n}+\mathbf{1}$ vesicles, of which one detaches towards the cytoplasm and so $\mathbf{n}$ vesicles remain.

From the probabilistic point of view, the two methods are independent events, that is, why their sum appears in the formula. Since the probability of formation and detachment of the vesicles depends on the concentrations of the substances on both sides of the membrane, the concentrations are proportionality factors. $\mathrm{K}$ is a one-dimensional constant. Thus, we obtained a system of differrential first degree equations, from which - knowing the limit conditions - we obtain the following expression for $\mathbf{n}=1$ :

$$
\mathbf{K} \cdot \frac{d P_{1}(t)}{d t}=\mathbf{c}_{\mathbf{k}} \cdot\left(\mathbf{P}_{\mathbf{0}}(\mathbf{t})-\mathbf{P}_{\mathbf{1}}(\mathbf{t})\right)+\mathbf{c}_{\mathbf{b}} \cdot\left(\mathbf{P}_{2}(\mathbf{t})-\mathbf{P}_{1}(\mathbf{t})\right)
$$

We cannot talk about pinocytosis unless there is at least one vesicle, that is why $c_{e} \cdot P_{0}(t)=0$, and $c_{i} \cdot P_{1}(t)$ does not make sense. For this reason, when $n=1$ these two terms disappear.

For the limit condition $n=M$ we will have:

$$
\mathbf{K} \cdot \frac{d P_{M}(t)}{d t}=\mathbf{c}_{\mathbf{k}} \cdot\left(\mathbf{P}_{M-1}(\mathbf{t})-\mathbf{P}_{\mathbf{M}}(\mathbf{t})\right)+\mathbf{c}_{\mathbf{b}} \cdot\left(\mathbf{P}_{M+1}(\mathbf{t})-\mathbf{P}_{M}(\mathbf{t})\right)
$$

This process allows the cell to take up large molecules, such as entire proteins, that find it difficult or impossible to cross the cell membrane, by other means. Foreign species, such as toxins, antigens, and pathogens, are also taken up in this way by cells of the immune system.

The most common kind of endocytosis is pinocytosis. Pinocytic vesicles, found in capillary wall, epithelia, and other cell types, are small and contain only solution. Certain cells, such as white blood cells, can form much larger vesicles, which contain solid matter as well; the internalization process in this case is called phagocytosis. [6]

\section{Crossed effects}

The biophysical models of crossed effects have not been elaborated too rigorously (detailed), this being the cause for which in the description of the processes found in the living organisms, there are bigger discrepancies between models and reality. [7]

The biophysical processes which actually characterize the biological systems can be better described by knowing the coupled transport processes. [8] In these systems the flows are not independent from one another, but they influence one another. Hence, in the living organisms, flows are not generated only by the conjugated generalized forces, but also by the simultaneous action of other forces. [9] Generally in the system there are as many flows as generalized forces and each force partially participated at the generation of each flow, so the simple types of the transport 
processes interfere with one another. It is extremely important the fact that the coupled transport systems can be reduced to the interconnected effect of the crossed effects.

\begin{tabular}{|c|c|c|}
\hline \multicolumn{3}{|c|}{ Type of the transport phenomenon } \\
\hline I. Simple & Flux & Generalized forces \\
\hline Diffusion & $\bar{M}$ & $\Delta \mathrm{c}$ \\
\hline Osmosis & $\mathrm{V}$ & $\Delta \mathrm{p}$ \\
\hline Viscosity & $\mathrm{P}$ & $\Delta \mathrm{v}$ \\
\hline $\begin{array}{l}\text { Thermal } \\
\text { conductibility }\end{array}$ & $\bar{E}$ & $\Delta \mathrm{T}$ \\
\hline $\begin{array}{l}\text { Electric } \\
\text { conductibility }\end{array}$ & $\mathrm{I}$ & $\Delta \varphi$ \\
\hline $\begin{array}{l}\text { II. Crossed effects (pairs): } \\
\Delta \mathrm{T} \Leftrightarrow \Delta \varphi ; \Delta \mathrm{T} \Leftrightarrow \Delta \mathrm{c} ; \Delta \mathrm{T} \\
\Delta \varphi \Leftrightarrow \Delta \mathrm{c} ; \Delta \mathrm{v} \Leftrightarrow \Delta \mathrm{c} ; \Delta \mathrm{T}\end{array}$ & $\begin{array}{l}\Leftrightarrow \Delta \mathrm{P} ; \Delta \mathrm{c} \\
\Leftrightarrow \Delta \mathrm{v} ; \Delta\end{array}$ & $\begin{aligned} & \Delta \mathrm{P} ; \Delta \varphi \Leftrightarrow \Delta \mathrm{P} ; \\
& \Delta \mathrm{v} ; \Delta \mathrm{P} \Leftrightarrow \Delta \mathrm{v} ;\end{aligned}$ \\
\hline
\end{tabular}

In most of the biological systems, more gradients act in general. For examples, in the case of the electrolytes, the concentration and the potential gradient cam function all the same. Several flows can coexists simultaneously. A certain type of flow is determined not only by the corresponding gradient, but basically it can be influenced by any force. In a simpler case, when there are two flows and two corresponding gradients, then, except for simpler transport phenomena, there are interactions between the existing flows and forces, namely the so called crossed effects.

\section{References:}

[1] Hoppe, W., Lohmann, W., Markl, H., Ziegler H.: Biophysics. Springer-Verlag, Berlin, 1983.

[2] Vincze, J.: The Transport Phenomena and Cross-effects in the Living Systems. NDP P., Budapest, 2001.

[3] Vincze, J.: Biophysics of the Apparatuses. NDP P. Budapest, 2020.

[4] Vincze, J.. Medical Biophysics. NDP P., Budapest , 2018.

[5] Kedem, O., Katchalsky, A. Mechanism of Active Transport. Trans. Faraday Soc. 1963, 59:1918-1930.

[6] Vincze, J., Vincze-Tiszay G.: Biophysical model of active transport through vesicles. Medical Research Archives, 2020, 8., (4):1-9.

[7] Sarson, E., Cobelli, C.: Modelling Methodology for Physiology and Medicine. Elsevier, 2014.

[8] Vincze, J.: The Biophysical Modeling of the Apparatuses in the Human Organism. Lambert Academic Publishing, Berlin, 2020.

[9] Vincze, J.: The Cross-effect in the Living Systems. Med. P. Budapest, 1984. 